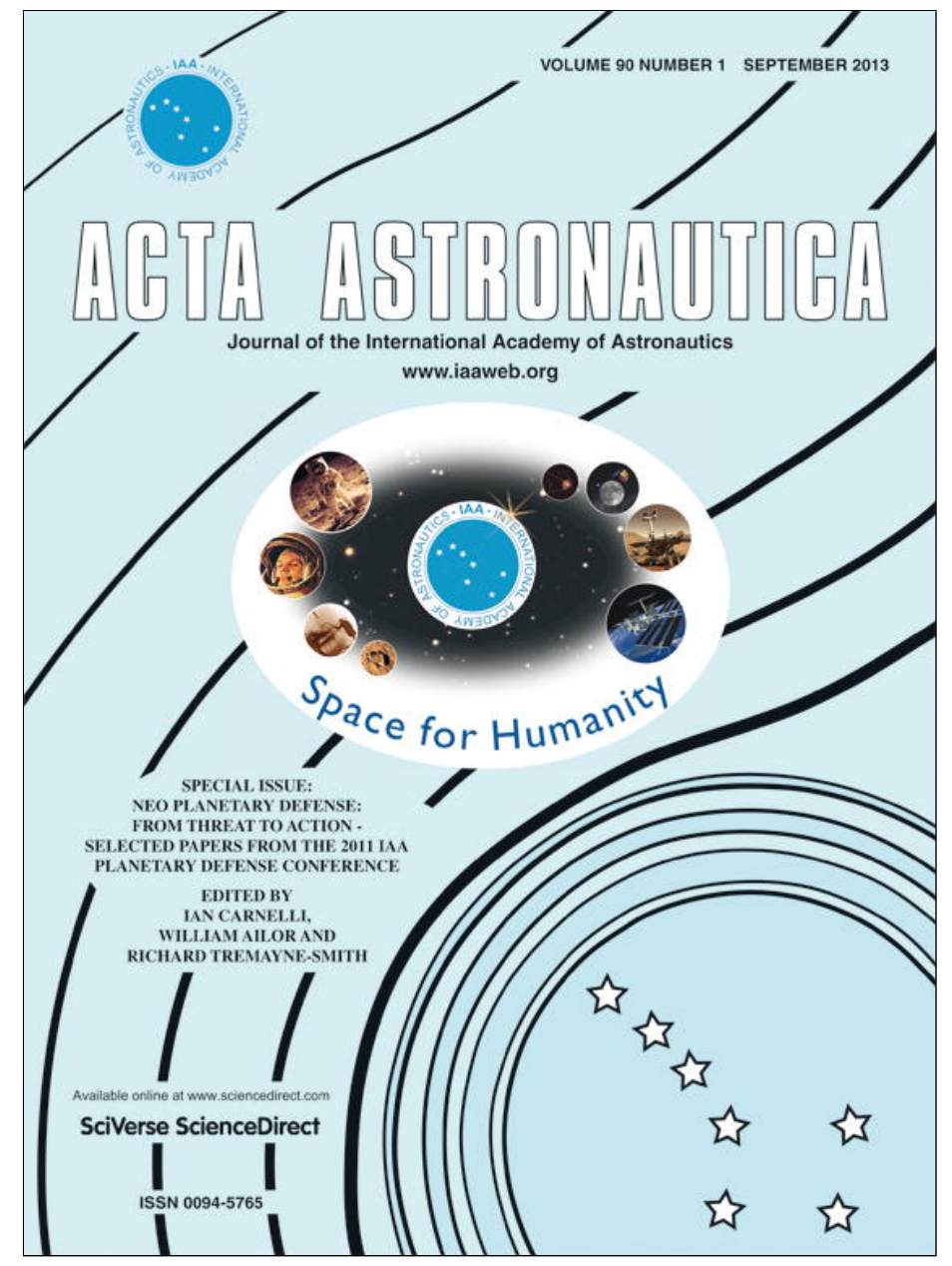

This article appeared in a journal published by Elsevier. The attached copy is furnished to the author for internal non-commercial research and education use, including for instruction at the authors institution and sharing with colleagues.

Other uses, including reproduction and distribution, or selling or licensing copies, or posting to personal, institutional or third party websites are prohibited.

In most cases authors are permitted to post their version of the article (e.g. in Word or Tex form) to their personal website or institutional repository. Authors requiring further information regarding Elsevier's archiving and manuscript policies are encouraged to visit: 


\title{
A space-based mission to characterize the IEO population
}

\author{
Ross Findlay ${ }^{\mathrm{a}, *}$, Olaf Eßmann ${ }^{\mathrm{a}}$, Jan Thimo Grundmann ${ }^{\mathrm{a}}$, Harald Hoffmann ${ }^{\mathrm{b}}$, \\ Ekkehard Kührt ${ }^{\text {b }}$, Gabriele Messina ${ }^{\text {b }}$, Harald Michaelis ${ }^{\text {b }}$, Stefano Mottola ${ }^{\text {b }}$, \\ Hartmut Müller ${ }^{a}$, Jakob Fromm Pedersen ${ }^{a}$
}

a DLR-Institute of Space Systems Robert-Hooke-Straße 7, 28359 Bremen, Germany

${ }^{\mathrm{b}}$ DLR-Institute of Planetary Research Rutherfordstraße 2, 12489 Berlin, Germany

\section{A R T I C L E I N F O}

\section{Article history:}

Received 18 January 2012

Received in revised form

30 May 2012

Accepted 4 August 2012

Available online 2 September 2012

\section{Keywords:}

Asteroid detection

Standardisation

Mission design

Small satellite

\begin{abstract}
A B S T R A C T
In 2007 the German Space Agency (DLR) initiated the Kompaktsatellit series of small satellites. With growing scientific interest in the threat of future asteroid impacts on Earth, the first mission selected for the Kompaktsatellit programme was AsteroidFinder, a mission to characterise the unknown Inner Earth Object (IEO) population. The mission is based around the AsteroidFinder Instrument (AFI), a high-performance optical telescope, with asteroids identified on-ground via their apparent motion against the fixed star background. Such a challenging mission implies significant demands on the Kompaktsatellit bus platform required to support the AFI. The tight constraints of small satellite design, namely time, finance and available mass, require innovative solutions to problems. With a launch scheduled for 2014 and the project due to enter Phase C in 2011, the challenges of achieving high science with a small satellite are already apparent.
\end{abstract}

(c) 2012 IAA. Published by Elsevier Ltd. All rights reserved.

\section{Introduction}

In 2007 the German Space Agency (DLR) initiated the Kompaktsatellit series of small satellites with a view to creating a small, flexible platform on which to base future advanced missions. With growing scientific interest in the threat of future asteroid impacts on Earth, the first mission selected for the Kompaktsatellit programme was AsteroidFinder, a mission to characterise the unknown Inner Earth Object (IEO) population in terms of mass, size and distribution. It is due to launch in 2014.

The mission is based around the AsteroidFinder Instrument (AFI), a high-performance optical telescope to be carried as the main payload on the inaugural mission. It shall operate for a period of one year, aiming to detect faint objects down to an apparent magnitude of $18.5 \mathrm{~V}_{\text {mag }}$ (under a sky background of $20.3 \mathrm{~V}_{\mathrm{mag}}$ ). Short duration fixed-location images shall be taken and stacked to form equivalent exposures, with a high image/slew duty-cycle allowing up

\footnotetext{
*Corresponding author. Tel.: +49421244201172.

E-mail address: ross.findlay@dlr.de (R. Findlay).
}

to 2880 square degrees of sky coverage per day. Asteroids shall then be identified on-ground via their apparent motion against the fixed star background in subsequent images.

Such a challenging mission implies significant demands on the satellite bus platform required to support the AFI. The tight constraints of small satellite design, namely time, finance and available mass, requires innovative solutions to problems. One particular challenge is to achieve the high pointing stability required for imaging while at the same time maintaining high slewing agility to maximise the useful science time. Furthermore, detection of such faint objects requires a compromise between stability, onboard processing power, and the available downlink scheme. It is the purpose of this paper to detail the current development status of the AsteroidFinder and the means by which such challenges are met.

\section{Mission overview}

The AsteroidFinder mission aims to characterise the population of Near Earth Objects (NEOs) and in particular 


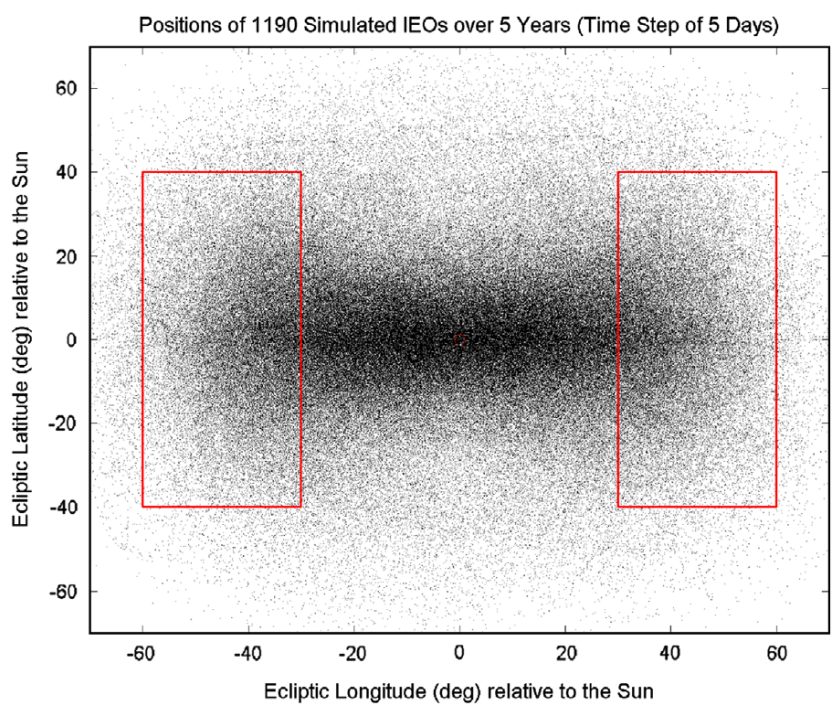

Fig. 1. AsteroidFinder region of interest (RoI).

Inner Earth Objects (IEOs) and Atens in terms of

- Number of objects.

- Orbital distribution and orbital parameters.

- Size distribution.

In order to characterise the population of IEO asteroids, the basic goal of AsteroidFinder is to observe and study a region of the sky ranging from $-60^{\circ}$ to $-30^{\circ}$ and $+30^{\circ}$ to $+60^{\circ}$ in ecliptic longitude and from $-40^{\circ}$ to $+40^{\circ}$ in ecliptic latitude (both relative to the Sun), as shown in Fig. 1.

Asteroids shall be recognisable through their motion on subsequent images taken by the AFI optical camera flying onboard the bus platform. The observations shall enable the determination of short-arc orbits, whose accuracy shall be adequate to recover the detected objects within one month from ground-based follow-up observations, or from AsteroidFinder itself.

In addition to detecting IEOs, AsteroidFinder should also discover a number of NEOs, and an even larger number of Main Belt Asteroids, thereby contributing to our understanding of those populations.

\section{Mission analysis}

Given the short mission duration of 1 year, optimal use must be made of the time spent in-orbit for observations of the RoI. As shown in Fig. 1, the Rol lies in two regions either side of the Sun (as seen from Earth). As such, the chosen orbit must avoid long eclipse periods whereby the satellite is behind the Earth and cannot view the RoI. A further constraint is given that a Low Earth Orbit must be used in order to minimise cost.

As such, a Sun Synchronous Orbit along the dawn-dusk terminator was selected. The orbit characteristics are defined in Table 1.

With this orbit, AsteroidFinder is able to observe within the Rol from every orbit over the entire year, with only limited eclipse times up to 26 min during worst-case seasons. A high degree of flexibility is also given as to
Table 1

Orbit characteristics (at launch).

\begin{tabular}{ll}
\hline Orbit & \\
\hline Orbit type & SSO \\
Altitude & $630 \mathrm{~km}$ \\
LTAN & $06: 00$ \\
Period & $\sim 97 \mathrm{~min}$ \\
Eclipse (worst-case) & $\sim 26 \mathrm{~min}$ \\
Inclination & $\sim 98^{\circ}$ \\
Orbit type & SSO \\
Altitude & $630 \mathrm{~km}$ \\
LTAN & $06: 00$ \\
Period & $\sim 97 \mathrm{~min}$ \\
\hline
\end{tabular}

which target area can be observed from any given orbital location as the effects of Earth-induced straylight are minimised.

The orbital height was selected in order to minimise the effects of atmospheric glow yet minimising the inorbit lifetime of the satellite after the end of mission (for compliance with space debris regulations). The minimum threshold for avoiding atmospheric glow is $550 \mathrm{~km}$; as such, a launch altitude of $630 \mathrm{~km}$ was selected.

\section{Operational concept}

A typical operational day for AsteroidFinder during the nominal science operation phase involves $14-15$ orbital revolutions around the Earth. The spacecraft will operate in a high image/slew duty cycle, alternately taking images of the night sky and slewing between subsequent images. Images shall be taken with an equivalent exposure time of approximately one minute, formed by real-time registered stacking of 300 individual exposures of $0.2 \mathrm{~s}$ duration to circumvent the blurring effects of the unintended spacecraft residual motion.

The majority of the operational day is spent in science operations, allowing for up to 720 images to be taken per day. Slew times between images are anticipated to be on the order of 1-2 min, depending on the observational scheme selected. The remainder of the nominal day entails four payload data downlink passes to Neustrelitz, with one daily pass acting simultaneously as a telemetry downlink/telecommand uplink pass with Weilheim. All operations are managed by the German Space Operations Centre (GSOC).

\section{Spacecraft requirements and design drivers}

Such a challenging mission implies tough constraints on the space segment that must be built to serve it, and this is especially so in the frame of a small satellite mission. The following details the main requirements and drivers on the space segment (i.e. spacecraft), many of which are not mutually exclusive

\subsection{High pointing stability}

In order to prevent blurring of the science images, it is essential that the spacecraft is as stable as possible during the individual exposures. Equivalent exposures must also 
show a great deal of stability to ensure maximal use of the given Field-of-View (FoV).

\subsection{High agility}

Available imaging time is one of the key drivers for maximising the science return from the mission: IEO detections can only be made from images, not slews. Hence, high spacecraft agility is required to minimise such scientific downtime. Note that high spacecraft agility is however typically at odds with a high pointing stability due to the destabilising effects of the more powerful actuators required to provide higher angular accelerations.

\subsection{Low-noise optical sensors}

The mission must be able to detect very faint objects amidst a non-negligible sky background: hence low-noise optical sensors must be used to prevent adding additional noise to the final images.

\subsection{Low distortion telescope}

In conjunction with the high stability demands on the spacecraft, the optical distortion of the AFI telescope must also be minimised to prevent blurring of images.

\subsection{On-board pre-processing for images}

The onboard register and stack process outlined for the science images requires considerable processing power. The high number of images to be stacked ( $\sim 300)$, coupled with the short time available for stacking (approximately $1 \mathrm{~min}$, i.e. before the next equivalent exposure sequence is begun), drives this demand for high-performance processing even further.

\subsection{Power availability}

The spacecraft has a relatively large power demand for a spacecraft in this class due to the high power required for image taking, high actuator cycling and downlink. This is particularly challenging given the variable Sun offset angle induced by the highly variable pointing scheme.

\subsection{Large payload volume}

A large payload volume is necessary to allow meeting the telescope distortion criterion without an excessively costly design; note that this conflicts with the high spacecraft agility requirement which favours a more compact design.

\subsection{Large payload data generation}

The science data cannot be highly compressed before use in the on-ground detection processes given that detections are based made on faint, sub-pixel events which are very close to the noise level. As such the resulting data volume that must be downloaded each day is unusually large for a small satellite (worst-case 720 images at 38 mebibytes (MiB) per image).

\subsection{Low detector temperature}

For detection at low apparent magnitudes with the available Charge Coupled Device technologies, stringently low temperatures must be guaranteed for the optical detectors to prevent adding additional noise.

\subsection{Straylight suppression}

The faint magnitude of objects to be detected requires strict straylight suppression, to ensure that only light sources from within the FoV of the telescope affect the final image.

\section{Space segment overview}

The AsteroidFinder space segment comprises of two elements: the AFI and the satellite bus. The AFI is responsible for all science duties, including the taking and preprocessing of science images. The satellite bus is responsible for providing all service needs to the AFI, including transporting of the AFI safely to orbit and maintaining the spacecraft health while operating. It also acts as the means of transferring all acquired science data to Earth, and as the sole interface to the Electrical Ground Support Equipment (EGSE) and the launch vehicle after integration of the AFI onto the bus.

An overview of the AFI is presented in Table 2, with the bus characteristics and performance summarised in Table 3.

A functional overview of the spacecraft is provided in Fig. 2. A detailed description of each subsystem follows.

\subsection{AsteroidFinder instrument (AFI)}

The AFI represents a TMA Cook-type optical telescope system with advanced Electron Multiplying Charge Coupled Device (EMCCD) detectors, complimented by a suite of onboard processors and supporting electronics. The telescope is composed of a monolithic Cesic $\AA$ structure, selected for its high thermal-mechanical stability and low mass, and is sized to make maximum use of the available envelope. EMCCD technology enables the use of image stacking due to their low read-out noise, thus allowing selection of short individual exposure times ( $\sim 0.2 \mathrm{~s})$ compatible with the maximum pointing stability of the spacecraft. The register and stack processing is done onboard in the AFI Data Processing Unit (DPU) to reduce the number of required images for downlink from 300 to 1 per equivalent exposure. Lossless compression will also be employed at this stage to reduce the required downlink volume further, without degrading the image quality.

\subsection{Attitude control system (ACS)}

The requirements placed on the ACS were amongst the most demanding for any part of the space segment. The conflicting demands for high stability and high agility lead to a highly optimised design using low-disturbance, high torque, actuators. For these purposes a standard four-wheel reaction wheel solution was adopted, with wheel offloading performed using magnetotorquers: propellant-based systems were ruled out early in the project due to their 
Table 2

AsteroidFinder instrument (AFI) performance and characteristics overview.

\begin{tabular}{|c|c|c|}
\hline AFI & & \\
\hline & Type & $\begin{array}{l}\text { Optical telescope with Electron Multiplying Charge Coupled Device (EMCCD) detectors and } \\
\text { digital processing electronics. }\end{array}$ \\
\hline & Mass & $40.4 \mathrm{~kg}$ (incl. margin) \\
\hline & Dimensions & $\begin{array}{l}\text { Complex shape telescope, focal plate and EMCCD electronics within an envelope of about } \\
500 \times 820 \times 767 \mathrm{~mm}^{3} \text {. Power supply and digital processing electronics accommodated in } \\
\text { spacecraft bus segment. }\end{array}$ \\
\hline & Power & On: $147 \mathrm{~W}$ (incl. margin) Standby: $78 \mathrm{~W}$ (incl. margin) \\
\hline & $\begin{array}{l}\text { Field of View } \\
\text { (effective) }\end{array}$ & $2^{\circ} \times 2^{\circ}$ \\
\hline & $\begin{array}{l}\text { Aperture } \\
\text { (effective) }\end{array}$ & $400 \mathrm{~cm}^{2}$ \\
\hline & Focal length & $76 \mathrm{~cm}$ \\
\hline & EMCCD pixel & $13 \mu \mathrm{m} \times 13 \mu \mathrm{m}$ \\
\hline & $\begin{array}{l}\text { Number of } \\
\text { pixels }\end{array}$ & $4 \times 1024 \times 1024$ \\
\hline & $\begin{array}{l}\text { Assumed data } \\
\text { per image }\end{array}$ & $38 \mathrm{MiB}$ (after $3 \times 3$ re-sampling and compression) \\
\hline
\end{tabular}

Table 3

AsteroidFinder satellite bus performance and characteristics overview.

\begin{tabular}{|c|c|c|}
\hline \multicolumn{3}{|l|}{ Satellite Bus } \\
\hline & Type & 3-axis stabilized, 3 degrees-of-freedom \\
\hline & Mass & $127 \mathrm{~kg}$ (incl. margin) \\
\hline & Dimensions & $1100 \times 811 \times 2230 \mathrm{~mm}^{3}($ stowed $)$ \\
\hline & Attitude control & Agility: $\sim 5^{\circ}$ in $1 \mathrm{~min}$. Absolute pointing error: $<120$ arcsec. Relative pointing \\
\hline & $\begin{array}{l}\text { system } \\
\text { Communications }\end{array}$ & $\begin{array}{l}\text { error: < } 0.875 \text { arcsec (individual AFI exposure). } \\
\text { Telecommand: }\end{array}$ \\
\hline & & S-Band (360 kiB per day) \\
\hline & & Telemetry: \\
\hline & & S-band (12.1 MiB per day) \\
\hline & & Payload data: \\
\hline & & X-band (28.4 GiB per day) \\
\hline & Command and & 48 GiB flash mass memory (beginning-of-life) \\
\hline & Mechanisms & Hinges and release mechanisms for solar arrays \\
\hline & Onboard & GPS receiver \\
\hline & $\begin{array}{l}\text { system } \\
\text { Power }\end{array}$ & $\begin{array}{l}\text { Unregulated bus: } 19-34 \mathrm{~V}, 2.1 \mathrm{~m}^{2} \text { fixed-deployable solar array } 517 \mathrm{~W} \text { and } \\
\text { maximum power generation } 1048 \mathrm{Wh} \text { battery capacity. }\end{array}$ \\
\hline & Structure & $\begin{array}{l}\text { Aluminium panel primary structure, milled from solid piece, with carbon fibre } \\
\text { payload segment. }\end{array}$ \\
\hline & Thermal & Mostly passive system electric heaters for survival cases \\
\hline
\end{tabular}

incompatibility with system goals (high cost, schedule impact, size, etc). The satellite is thus a three degree of freedom, three-axis stabilised solution. A conventional control loop is used (see Fig. 3.) with the AFI telescope incorporated into the control loop as an additional rate sensor. This is necessary to meet the stability requirements without additional sensors. Fast slews (on the order of $5^{\circ}$ in $1 \mathrm{~min}$ ) are made possible via an optimised control law.

For nominal operations, fast reorientations between two inertially fixed target locations via a time-optimised slew are the standard; for downlink, due to the high Moments-of-Inertia (MoIs) of the spacecraft (thus rendering ground-based target pointing unfeasible) nadir pointing is used.

The ACS must also monitor and maintain a comprehensive set of attitude constraints imposed by the Sun and Earth, and be able to generate warning signals to the AFI whenever the Sun, Earth or Moon is likely to enter the FoV of the telescope. This is essential to safeguard the health of the sensitive AFI detectors.

\subsection{Electrical power system (EPS)}

A nominal spacecraft power demand in the range of 250-350 W, coupled with the need to limit deployed solar array area (for spacecraft agility purposes, see below), lead to the focus on high-efficiency in the EPS. High performance triple-junction solar cells (28\% efficiency class) are used along with Maximum Power Point Tracking devices to extract the highest amount of power from the available solar array area. Furthermore, efficient localised power conversion is used to reduce losses in the system, with all 


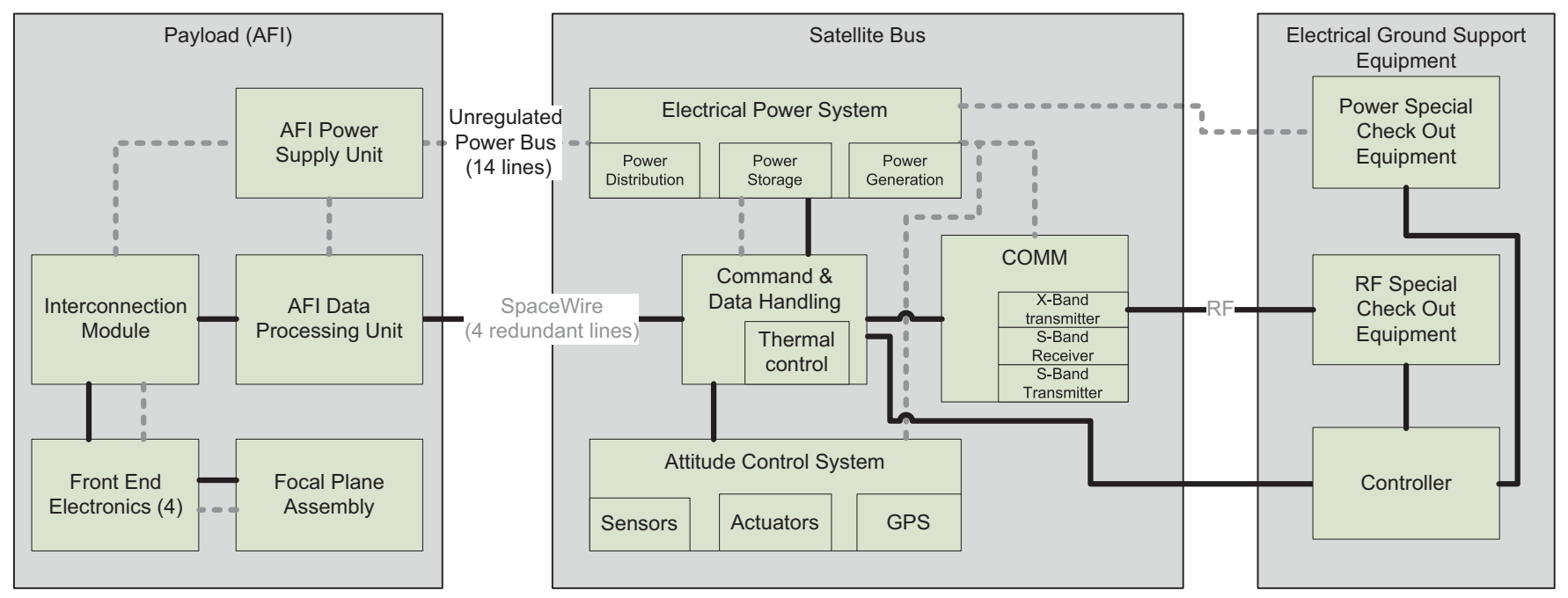

Fig. 2. Functional overview of AsteroidFinder space segment (note red lines indicate power connections and green lines indicate data interfaces). (For interpretation of the references to colour in this figure legend, the reader is referred to the web version of this article.)

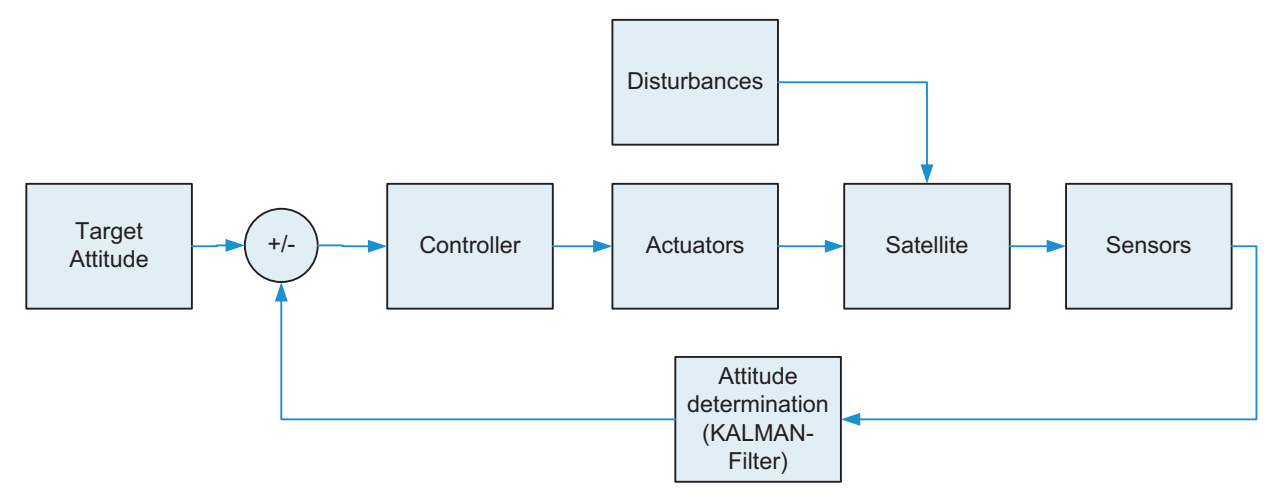

Fig. 3. ACS control loop.

distribution distances kept as short as possible. A large battery $(\sim 1400 \mathrm{Wh})$ completes the design, necessary to fulfil all Launch and Early Operations Phase (LEOP) obligations.

The power subsystem is also charged to provide a safe hardwired switch-off/switch-on functionality to aid with the recovery of the spacecraft in the event of a total power outage.

\subsection{Command and data handling (CEDH)}

A novel network-oriented approach is used for the onboard C\&DH subsystem, allowing the desire for high system-level reliability and dependability to be met with non-space qualified parts. A central connection node, known as the Middleware Switch (MWS), acts essentially as a network router between all units (see Fig. 4) to manage communications [1]. On top of this, networkoriented software provides a similarly standardised ability to communicate between distributed software tasks without depending upon direct interfaces to these other systems (see Fig. 5). Such a concept eases the risk of integrating additional components and software modules into the system at a later date, as only the interface

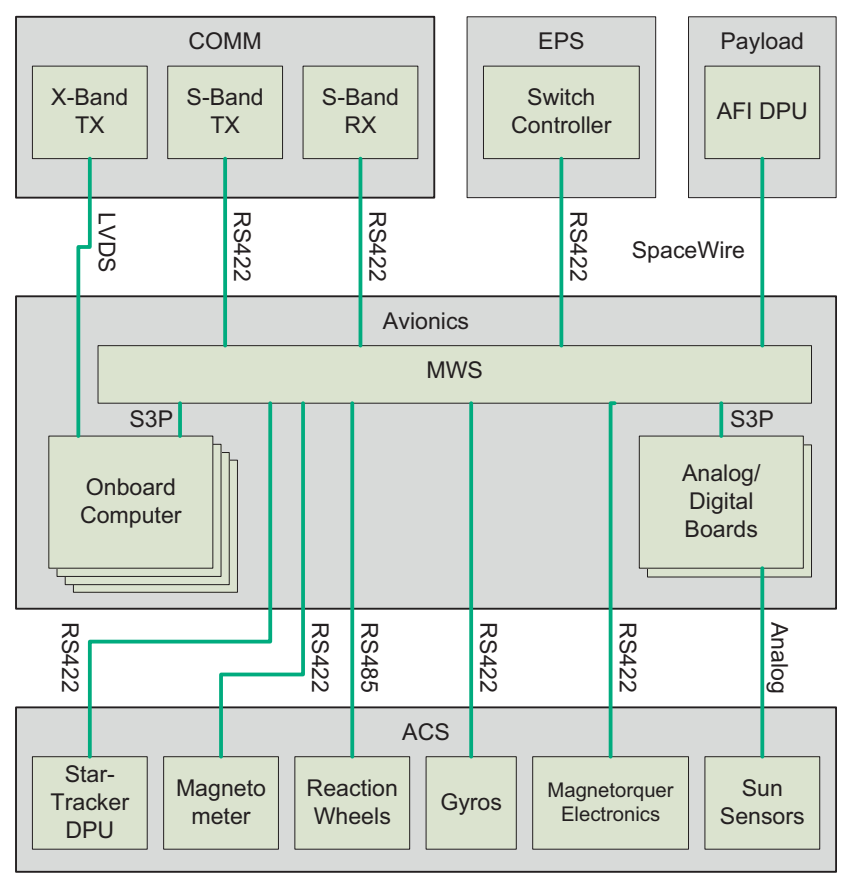

Fig. 4. Middleware Switch (MWS) concept overview. 


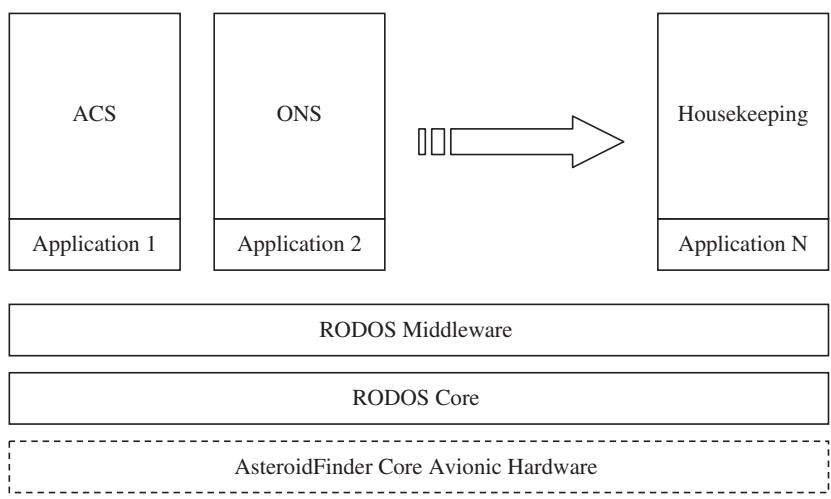

Fig. 5. RODOS middleware (software) concept overview.

between the new component and the Middleware needs to be considered: all other interfaces are independent.

Supplementing the Middleware is the Real-time Operating System (RODOS) running on the C\&DH board (the central processing node of the C\&DH subsystem). RODOS provides resource management, thread synchronisation and communication, and input/output and interrupts management to all other RODOS applications. It represents a realtime embedded operating system for applications demanding high dependability, using priority-based scheduling and round robin for same priority threads, and a publisher/ subscriber protocol for asynchronous message exchange.

The C\&DH board, besides running the RODOS Core, also provides the mass memory for storage of all science data before downlink. Four C\&DH boards are incorporated into the design, with the nominal worker board being shadowed by a hot redundant monitor board. This ensures reliable monitoring of the health and functionality of the nominal board at all times, and a quick handover in the event of a failure. The other two boards are kept in cold redundancy in case of the permanent failure of either nominal board.

\subsection{Rf Communications}

The large downlink requirement for the science data leads to the adoption of a split system for spacecraft downlinks: nominal telemetry, to be compatible with the selected ground stations at Weilheim and Neustrelitz, is to be downlinked once per day in S-band; science data, with its much higher data volume, shall be downlinked via four daily passes to Neustrelitz in X-band. All telecommanding of the spacecraft (from Weilheim) will also be in S-band.

The S-band reception chain is omni-directional and always active to ensure commandability of the spacecraft in any state. For telemetry downlink, two antennae configurations are possible: while the omni-directional telecommanding network is available as backup, a dedicated nadirpointed antenna will be used as standard to strengthen the broadcast during routine operations. For the X-band downlinks, due to the inability of following a ground-pointing slew scheme (due to insufficient spacecraft agility, see above), a range-compensated, nadir-pointing antenna will be used.

\subsection{Navigation}

A Global Positing System (GPS) receiver and onboard navigation software are utilised in order to provide accurate time and position information for each science image. The navigation software is also essential for propagating the location of celestial objects such as the Earth and Moon which are required as part of the attitude constraints monitoring by the ACS (see above).

A flight proven Commercial Off-the-Shelf (COTS) design is used with heritage from missions including PRISMA and PROBA-2 [2,3]. Such a solution is specifically tailored for the low mass/power requirements of small satellite missions.

\subsection{Mechanical design}

The main drivers for the AsteroidFinder structural design are to provide a large payload volume for the AFI that is desensitized to thermal instability, while minimising the spacecraft MoIs in order to maximise the agility.

The spacecraft accommodation is split into two main segments: the bus compartment and the payload compartment (housing the AFI) (see Fig. 6). The bus compartment consists of an aluminium box with an integrated hash structure to attach most of the bus units. This structure provides a solid load path during launch while maximising the available volume for unit placement and attachment. Heavy units such as the batteries are placed as close as possible to the centreline of the spacecraft, in order to minimise the Mols. The payload compartment contains a carbon fibre frame structure, used for its low mass benefits; the upper triangle of which acts as a sunshield to protect the AFI aperture from direct sunlight during nominal operations. The spacecraft also has a large

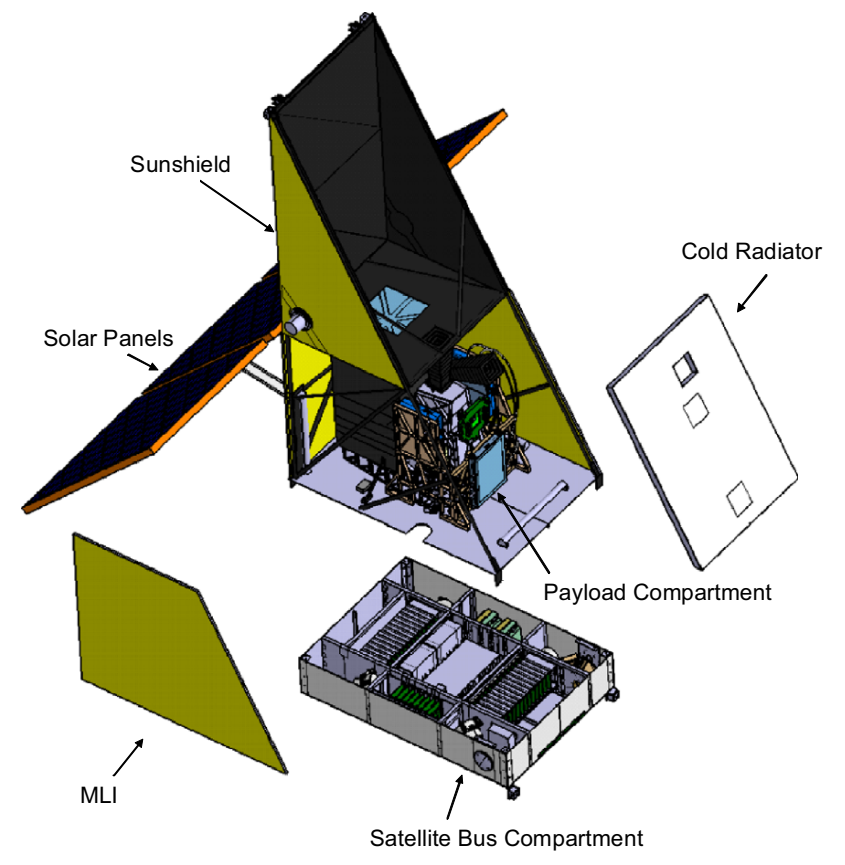

Fig. 6. Spacecraft configuration. 
low temperature radiator attached in the sunshield plane to ensure a constant cold face to deep space.

The solar arrays are pointed to the Sun with a $45^{\circ}$ offset (to maximise power generation at the centreline of the RoI) and are deployed via "tape-spring" passive deployment hinges (see Fig. 7). These hinges use two semi-circular elements, which, when folded, store mechanical energy via the deformation of the elements along the folding crease. When the appropriate release mechanisms are deployed, the hinges straighten out in a manner similar to a folded measuring tape. Glass Fibre Reinforced Polymer is used for the hinges due to its high deformability and high stiffness.

\subsection{Thermal control system (TCS)}

The TCS must maintain all spacecraft units within their nominal temperature ranges for all phases of the mission. It is driven primarily by the stringent requirements of the AFI detectors, with a low and narrow range of between $-100{ }^{\circ} \mathrm{C}$ and $-85^{\circ} \mathrm{C}$ when active (at the interface). Such a low temperature leads to the reservation of much of the available external bus area for a specific low-temperature radiator (see above). By placing constraints on the allowable spacecraft attitudes such that this face is always pointed towards deep space, this low temperature can be achieved. The AFI is thermally decoupled from the rest of

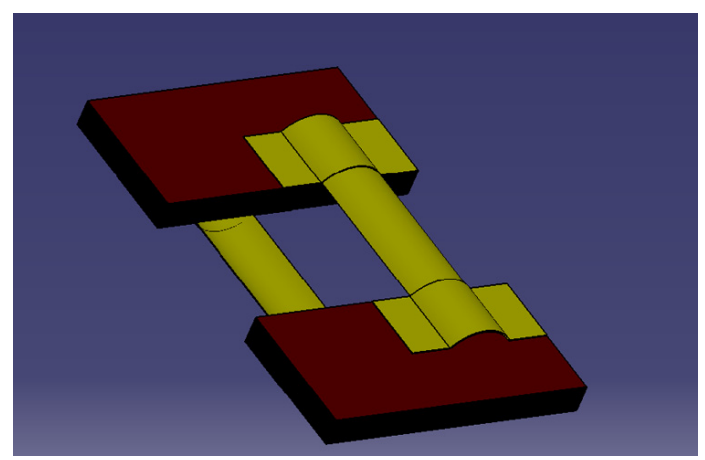

Fig. 7. Tape-spring deployment hinges. the spacecraft via titanium mounts and low-emissivity coatings to reduce competing heat-leaks.

All other thermal requirements are met by conventional passive means, using internal distribution of heat and white-painted radiators to reduce temperatures and to ensure stability. The high mean power consumption of the spacecraft necessitates the use of the majority of external surface areas as radiators; MLI is also used to prevent solar irradiation from heating the internal components on all other faces. Emergency heaters will be used on critical units to prevent overcooling, with all heater setpoints adjustable from ground to allow for tailoring as the mission progresses.

\section{Unit development}

To reduce project cost and development time, significant use has been made of Commercial-Off-The-Shelf (COTS) parts. This has allowed much of the development effort to focus only on those areas which are mission specific and cannot be easily procured as standard parts. While this does engender some additional risk for those COTS parts which are not fully space qualified, this is abated through the use of sufficient unit redundancy and design margins.

Fig. 8 provides a graphical overview of the use of COTS technology in the aforementioned subsystems. Further discussion is provided in [4].

\section{Conclusion}

Despite significant demands and constraints upon the system, it is shown that the challenges of achieving highquality science with a small satellite can be met. Importantly, it needs to be noted that a small satellite does not necessarily represent a small mission: for instance, by following an uncharacteristically intense downlink scheme, a daily downlink capacity comparable to many larger scale missions has been demonstrated. Balancing of resources, for instance by

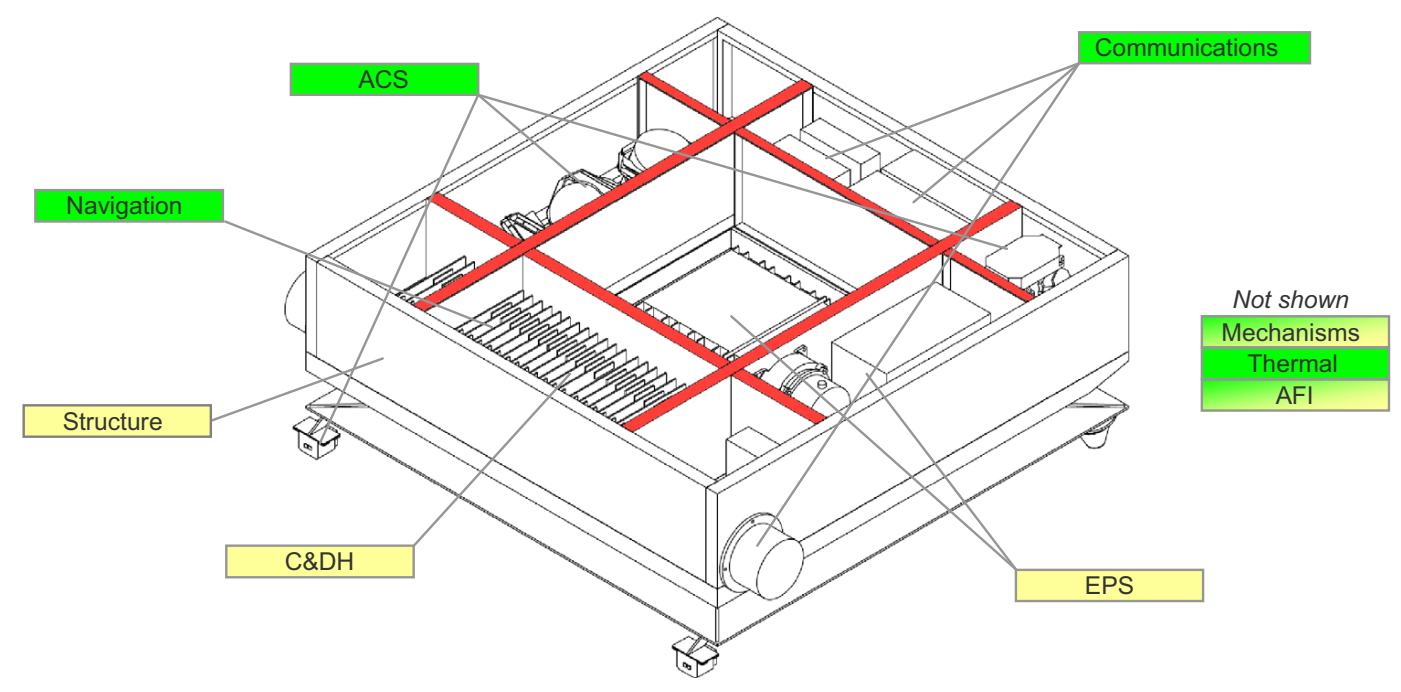

Fig. 8. COTS usage in AsteroidFinder (note that green indicates full COTS procurement, yellow indicates an in-house development). (For interpretation of the references to colour in this figure legend, the reader is referred to the web version of this article.) 
selection of COTS parts where possible, allows focussing the development effort on the key driver for the mission: the AFI. Willingness to employ novel technologies, for instance in the "tape-spring" mechanical deployment devices, AFI Cesic $\AA$ structure and AFI EMCCDs allow for the pushing of spacecraft performance to the maximum. By employing modular design and reliable system-level concepts (in particular via the C\&DH middleware-based concept), project risk can also be minimised by accounting for non-space qualified parts and late changes to the design.

\section{Acknowledgements}

The author would like to acknowledge the significant contribution of all members of the AsteroidFinder satellite bus design team not named above: Volodya Baturkin; Frank Dannemann; Martin Drobczyk; Ebrahim Haririan; Ansgar Heidecker; Elnaz Kheiri; Horst-Georg Lötzke; Olaf Maibaum; Markus Markgraf; Olaf Mierheim; Falk Nohka; Malak
Samaan; Jan-Carsten Schröder; Martin Siemer; Andrzej Sonnek; Marco Straubel; Paul Swatschina; Nawarat Termtanasombat; Carl Johann Treudler.

\section{References}

[1] S. Montenegro, E. Haririan, A fault-tolerant middleware switch for space applications, in: Proceedings of the Third IEEE International Conference on Space Mission Challenges for Information Technology, Pasadena, 19-23 July 2009.

[2] J.S. Ardaens, S. D'Amico, O. Montenbruck, Flight results from the PRISMA GPS-based navigation, in: Proceedings of the 5th ESA Workshop on Satellite Navigation Technologies, Noordwijk, 8-10 December 2010.

[3] O. Montenbruck, J.L. Issler, M. Markgraf, F. Mercier, S. Santandrea, A. Garcia, J. Naudet and S. Serre, GPS-Based Precise orbit determination and real-time navigation of the PROBA-2 spacecraft, in: Proceedings of the 5th ESA Workshop on Satellite Navigation Technologies, Noordwijk, 8-10 December 2010.

[4] R. Findlay, O. Eßmann, H. Hoffmann, G. Messina, S. Mottola, H. Müller, J.F. Pedersen, AsteroidFinder: implementing a small satellite mission to detect IEO's, in: Proceedings of the 62nd International Astronomical Congress, Cape Town, 3-7 October 2011. 